\title{
Diagnóstico da sustentabilidade de propriedades de agricultores familiares: uma aplicação do método IDEA
}

O debate em torno do modelo "produtivista" tem levado a constantes questionamentos sobre seus impactos, o que tem reforçado uma nova orientação de agricultura que não seja considerada apenas por seu papel fornecedora de alimentos, mas também por suas funções sociais, econômicas e ambientais, dentro de uma perspectiva de paisagem e conservação. Recentemente, estudos têm buscado analisar a sustentabilidade de agroecossistemas a partir de métodos que utilizam indicadores com capacidade de monitorar as práticas e técnicas agrícolas, heranças culturais, situação econômica e aspectos sociais. Dessa forma, este artigo objetivou avaliar o nível de sustentabilidade ambiental de propriedades rurais familiares que estão em transição agroecológica no Agreste Meridional de Pernambuco. Foram avaliadas a sustentabilidade de dez propriedades rurais familiares a partir do método IDEA, versão 4 (IDEA v4). Os agroecossistemas avaliados apresentaram uma média de 74,8\% para dimensão Sócio-territorial, 79,3\% para dimensão Agroecológica e 65,1\% para dimensão Econômica, sendo as melhores pontuações relativas aos aspectos ambientais e as piores ao fator econômico. Assim, chama-se atenção para a dimensão Econômica, tendo em vista a necessidade de melhorar algumas potencialidades e buscar reduzir as limitações ao longo do espaço e do tempo. $O$ uso de indicadores de sustentabilidade pode colaborar na autogestão de agroecossistemas, tanto para técnicos quanto para agricultores e ainda contribuir na operacionalização de ferramentas que visam a sustentabilidade e tomada de decisão de gestores e políticas públicas.

Palavras-chave: Agroecologia; Desenvolvimento Sustentável; Sistemas agrícolas.

\section{Diagnosis of the sustainability of family farmers' properties: an application of the IDEA method}

The debate around the "productivist" model has led to constant questions about its impacts, which has reinforced a new orientation for agriculture that is not only considered for its role as a food supplier, but also for its social, economic and environmental functions, within a landscape and conservation perspective. Recently, studies have sought to analyze the sustainability of agroecosystems using methods that use indicators with the capacity to monitor agricultural practices and techniques, cultural heritage, economic situation and social aspects. Thus, this article aimed to assess the level of environmental sustainability of family farms that are in agroecological transition in Agreste Meridional de Pernambuco. The sustainability of ten family farms was assessed using the IDEA method, version 4 (IDEA v4). The evaluated agroecosystems presented an average of $74.8 \%$ for the Socio-territorial dimension, $79.3 \%$ for the Agroecological dimension and $65.1 \%$ for the Economic dimension, with the best scores relating to the environmental aspects and the worst to the economic factor. Thus, attention is drawn to the Economic dimension, in view of the need to improve some potentialities and seek to reduce limitations over space and time. The use of sustainability indicators can collaborate in the self-management of agro-ecosystems, both for technicians and for farmers, and also contribute to the operationalization of tools aimed at sustainability and decision making by managers and public policies.

Keywords: Agroecology; Sustainable development; Agricultural systems.

Topic: Extensão e Desenvolvimento Rural

Reviewed anonymously in the process of blind peer.

Horasa Maria Lima da Silva Andrade (iD

Universidade Federal do Agreste de Pernambuco, Brasil http://lattes.cnpq.br/4314101991387960

http://orcid.org/0000-0002-5366-6610

horasaa@gmail.com

Romário Nunes da Silva (iD

Universidade Federal do Agreste de Pernambuco, Brasil

http://lattes.cnpq.br/8990193402311901

http://orcid.org/0000-0002-0401-8000

romario.nuness@gmail.com

\section{Luciano Pires de Andrade (it)}

Universidade Federal do Agreste de Pernambuco, Brasil

http://lattes.cnpq.br/5367851306746294

http://orcid.org/0000-0001-5818-711X

lucianopandrade@gmail.com
Received: 06/04/2021

Approved: 02/05/2021
Referencing this:

ANDRADE, H. M. L. S.; SILVA, R. N.; ANDRADE, L. P.. Diagnóstico da sustentabilidade de propriedades de agricultores familiares: uma aplicação do método IDEA. Revista Ibero Americana de Ciências Ambientais, v.12, n.5, p.39-48, 2021. DOI:

http://doi.org/10.6008/CBPC2179-6858.2021.005.0004 


\section{INTRODUÇÃO}

No sentido do desenvolvimento sustentável, a contribuição das atividades da agricultura frequentemente é questionada por seus efeitos ambíguos, seja pela exploração dos recursos naturais, como também, pela contribuição com a prestação de serviços não mercantis (multifuncionalidade) que vão além da produção de alimentos. O modelo agrícola vigente, tido como "produtivista", e "intensivo" tem implicado em sistemas de produção com forte uso de insumos químicos (produtos fitossanitários e fertilizantes minerais), de agrotóxicos e mecanização do manejo, acarretando num desprendimento das potencialidades naturais do meio ambiente. Ao favorecer a produtividade, este modelo é responsável por inúmeros impactos negativos sobre o meio ambiente como a qualidade e recursos quantitativos das águas superficiais e profundas, contaminação do solo e água, e a diminuição das áreas florestadas e biodiversidade (PUJOL et al., 1999).

Este processo ocorrido também tem transformado as paisagens rurais, e por isso que, os danos causados pela agricultura se tornaram objetos de preocupação por muitos atores, o que tem incentivado agricultores a adaptarem a atividade produtiva, levando em conta as novas demandas da sociedade que requer padrões e práticas de agriculturas sustentáveis. Os consumidores tornaram-se mais atentos em relação aos meios de produção, buscando cada vez mais produtos que causem menos danos ecológicos e riscos à saúde (TAUBER, 2007). O debate levantado sobre novos padrões de produção e consumo, reforçou uma nova orientação de agricultura que não seja considerada apenas por seu papel de fornecedora de alimentos, mas também por suas funções de amenizadora de danos e de serviços ambientais, sociais e políticos (soberania alimentar). Todavia, essa demanda da sociedade pelo desenvolvimento de atividades agrícolas alicerçadas na agricultura sustentável levanta questionamentos acerca de ações individuais ou coletivas, modelos de transição para novos sistemas agrícolas, estratégias de desenvolvimento territorial e meios de condução para agricultura sustentável (ZAHM et al., 2015).

Recentemente, ao pesquisarem sobre maneiras de avaliar a sustentabilidade agrícola, Schader et al. (2014), Lairez et al. (2015), De Olde et al. (2016) e Zahm et al. (2018) identificaram uma grande variedade de ferramentas e métodos, no qual, abrange uma diversidade de critérios e indicadores. Mesmo concordando que o estabelecimento de uma estrutura de avaliação é o primeiro passo em qualquer análise agrícola, para realizar esse processo faz-se necessário verificar alguns pontos e métodos. Dentre os pontos, encontram-se: os objetivos gerais do método em questão; discussão da literatura teórica e as visões subjacentes dos autores em relação à sustentabilidade; caracterização da unidade agrícola avaliada; identificação das relações com outros sistemas; e o estabelecimento dos procedimentos a serem utilizados (HANSEN, 1996; LÓPEZ-RIDAURA et al., 2002; POPE et al., 2004; KATES et al., 2005; LÓPEZ-RIDAURA et al., 2005).

Dentre os vários tipos de métodos que visam avaliar o nível de sustentabilidade de um sistema de produção agrícola, o Indicateurs de Durabilité d'une Exploitation Agricole ou Indicadores de Desenvolvimento Sustentável das Explorações Agrícolas (IDEA) se destaca ao apresentar uma abordagem baseada no uso indicadores. Esse método foi desenvolvido na França, no final da década de 1990, e foi 
atualizado em algumas ocasiões (VILAIN et al., 2008; ZAHM et al., 2015). Portanto, diante disto, este artigo objetivou avaliar o nível de sustentabilidade ambiental de propriedades rurais familiares que estão em transição agroecológica, localizadas no Agreste Meridional de Pernambuco, a partir do método IDEA, que é tão pouco usual nesta região e sua aplicação pode subsidiar atualizações.

\section{METODOLOGIA}

\section{Área de estudo}

Entre março de 2019 e setembro de 2020 foram avaliadas dez propriedades rurais familiares que estão em transição agroecológica: Agroecossistema 1 (AG1), Agroecossistema 2 (AG2), Agroecossistema 3 (AG3), Agroecossistema 4 (AG4), Agroecossistema 5 (AG5), Agroecossistema 6 (AG6) no município de Garanhuns; Agroecossistema 7 (AG7) - no município de Saloá; Agroecossistema 8 (AG8) no município de Bom Conselho; Agroecossistema 9 (AG9) e Agroecossistema 10 (AG10) no município de Jucati. Todos os municípios estudados pertencem ao território do Agreste Meridional (Figura 1), que está geograficamente localizado na Mesorregião do Agreste e do Sertão Pernambucano, sendo uma área intermediária entre a Mata e o Sertão, com extensão total de $13.153 \mathrm{~km}^{2}$ e envolve as Microrregiões do Vale do Ipanema, Vale do Ipojuca, Garanhuns e Sertão do Moxotó. Seu clima e o relevo são alguns dos maiores diferenciais em relação ao resto do Estado, propiciando, além da atividade pecuária, diversidade de cultivos, a exemplo da floricultura e oferecendo diversas opções de turismo, atividades econômicas dinâmicas e empregadoras de mão-de-obra local. Também é conhecida como bacia leiteira do Estado, tendo como base econômica a pecuária leiteira, com a produção de leite e derivados de forma artesanal e industrial (BRASIL, 2011).

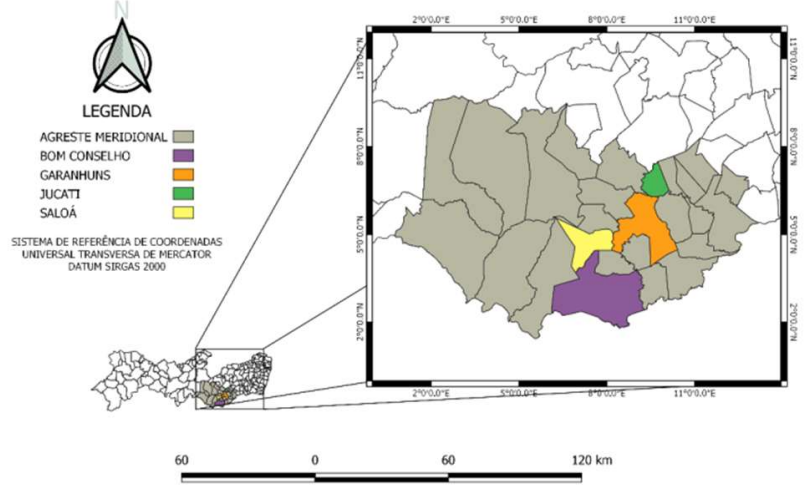

Figura 1: Localização dos municípios de Bom Conselho, Garanhuns, Jucati e Saloá, no Agreste Meridional de Pernambuco.

\section{Procedimentos metodológicos}

Foi realizada uma avaliação qualitativa a partir do método IDEA que se baseia na possibilidade de avaliar a sustentabilidade de um sistema agrícola através da quantificação de características técnicas, espaciais, econômicas e humanas, e das práticas julgadas favoráveis aos meios biofísico e social (VILAIN, 2008). Foi aplicada a versão 4 (IDEA v4), pois permite uma caracterização no nível sistêmico a partir de uma abordagem teórica baseada nas propriedades de sistemas agrícolas sustentáveis. Esta define a agricultura sustentável como economicamente viável, ecologicamente correta, socialmente justa e humana. A sua 
aplicação está abordada e estruturada na agregação de 53 indicadores que abrangem as três dimensões do Desenvolvimento Sustentável (Agroecológico, Sócio-territorial, Econômico) e amparada nos cinco atributos dos sistemas agrícolas sustentável (autonomia; robustez; capacidade produtiva e reprodução de bens e serviços; ancoragem territorial e responsabilidade geral) (ZAHM et al., 2019).

A classificação está de acordo com um sistema de unidades de sustentabilidade de acordo com uma escala adaptada a cada indicador. Os indicadores estão classificados entre zero e um valor máximo de sustentabilidade. A pontuação de cada componente decorre da soma das pontuações de cada um de seus indicadores, com um valor máximo de 20 a 35. A pontuação máxima para cada dimensão é 100 unidades correspondentes ao nível mais alto de durabilidade (Quadro 1). A pontuação final de sustentabilidade da propriedade avaliada permite ao agricultor identificar onde estão localizadas suas margens ou sua maior necessidade de progresso (ZAHM et al., 2019). Ressalta-se a ocorrência de uma adaptação dos indicadores à região avaliada, sem comprometer os princípios científicos de sua construção. A coleta dos dados se deu por questionários estruturados com o público alvo. Este tipo de pesquisa é comumente utilizado para a obtenção de dados ou informações sobre características, ações ou opiniões de um determinado grupo de pessoa (survey) (PINSONNEAULT, 1993).

Quadro 1: Indicadores valorados para avaliação da sustentabilidade de acordo com as 3 principais dimensões: Sócio-territorial, Agroecológica e Econômica.

\begin{tabular}{|c|c|c|c|c|}
\hline Dimensão & Componente & Indicador & $\begin{array}{l}\text { Pontuação } \\
\text { máxima }\end{array}$ & Teto \\
\hline \multirow[t]{24}{*}{ Sócio-territorial } & \multirow[t]{5}{*}{ Alimentação } & Produção de alimentos na propriedade & 6 & \multirow[t]{5}{*}{25} \\
\hline & & Contribuição para o equilíbrio alimentar global & 6 & \\
\hline & & Qualidade da produção de alimentos & 6 & \\
\hline & & Perdas e desperdícios & 6 & \\
\hline & & Ligações sociais, heranças e culturas alimentares & 6 & \\
\hline & \multirow{8}{*}{$\begin{array}{l}\text { Desenvolvimento local } \\
\text { e economia circular }\end{array}$} & Compromisso com abordagens ambientais territoriais & 5 & \multirow[t]{8}{*}{25} \\
\hline & & Serviços de mercado no território & 3 & \\
\hline & & Promoção de produtos por canais curtos ou próximos & 5 & \\
\hline & & Desenvolvimento de recursos locais & 5 & \\
\hline & & $\begin{array}{l}\text { Avaliação e qualidade do patrimônio (construções, paisagem, } \\
\text { conhecimento local e recursos naturais) }\end{array}$ & 3 & \\
\hline & & Acessibilidade do espaço & 3 & \\
\hline & & Gestão de resíduos não orgânicos & 3 & \\
\hline & & Redes de inovação & 3 & \\
\hline & \multirow{5}{*}{$\begin{array}{l}\text { Emprego e qualidade } \\
\text { no trabalho }\end{array}$} & Contribuição para o emprego e gestão de funcionários & 6 & \multirow[t]{5}{*}{25} \\
\hline & & Trabalho coletivo & 6 & \\
\hline & & Intensidade e qualidade no trabalho & 6 & \\
\hline & & Casa, higiene e segurança & 5 & \\
\hline & & Assistência técnica & 5 & \\
\hline & \multirow{5}{*}{$\begin{array}{l}\text { Ética e } \\
\text { desenvolvimento } \\
\text { humano }\end{array}$} & Envolvimento social territorial e solidariedade & 6 & \multirow[t]{5}{*}{25} \\
\hline & & Processo de transparência & 6 & \\
\hline & & Qualidade de vida & 6 & \\
\hline & & Isolamento & 6 & \\
\hline & & Bem-estar animal & 6 & \\
\hline & & Total & 118 & 100 \\
\hline \multirow[t]{13}{*}{ Agroecológica } & \multirow[t]{5}{*}{ Diversidade funcional } & Diversidade de espécies cultivadas & 5 & \multirow[t]{5}{*}{20} \\
\hline & & Diversidade genética & 5 & \\
\hline & & Diversidade temporal de culturas & 5 & \\
\hline & & Qualidade da organização espacial & 5 & \\
\hline & & Gestão da biodiversidade & 5 & \\
\hline & \multirow{3}{*}{$\begin{array}{l}\text { Fluxo de materiais e } \\
\text { energia através de } \\
\text { uma busca por } \\
\text { autonomia }\end{array}$} & Autonomia em energia, materiais, equipamentos, sementes e plantas & 8 & \multirow[t]{3}{*}{20} \\
\hline & & Autonomia alimentar da propriedade rural & 8 & \\
\hline & & Autonomia de nitrogênio & 8 & \\
\hline & \multirow{3}{*}{$\begin{array}{l}\text { Sobriedade no uso dos } \\
\text { recursos }\end{array}$} & Sobriedade no uso da água e compartilhamento do recurso & 8 & \multirow[t]{3}{*}{20} \\
\hline & & Sobriedade no uso do fósforo & 8 & \\
\hline & & Sobriedade no consumo de energia & 8 & \\
\hline & \multirow{2}{*}{$\begin{array}{l}\text { Garantia das condições } \\
\text { favoráveis de }\end{array}$} & Raciocinando o uso da água & 8 & \multirow[t]{2}{*}{20} \\
\hline & & Promoção da fertilidade do solo & 8 & \\
\hline
\end{tabular}




\begin{tabular}{|c|c|c|c|c|}
\hline & \multirow{2}{*}{$\begin{array}{l}\text { produção a médio e } \\
\text { longo prazo }\end{array}$} & Proteção da saúde de culturas e animais & 4 & \\
\hline & & Garantia dos meios materiais de produção & 4 & \\
\hline & \multirow{4}{*}{$\begin{array}{l}\text { Redução dos impactos } \\
\text { na saúde humana e } \\
\text { nos ecossistemas }\end{array}$} & Redução do impacto das práticas na qualidade da água & 6 & \multirow[t]{4}{*}{20} \\
\hline & & Redução do impacto das práticas na qualidade do ar & 6 & \\
\hline & & Redução do impacto das práticas nas mudanças climáticas & 6 & \\
\hline & & Redução do uso de produtos fitossanitários e veterinários & 6 & \\
\hline & \multicolumn{2}{|r|}{ Total } & 121 & 100 \\
\hline \multirow[t]{12}{*}{ Econômica } & \multirow{3}{*}{$\begin{array}{l}\text { Viabilidade econômica } \\
\text { e financeira }\end{array}$} & Capacidade econômica & 20 & \multirow[t]{3}{*}{35} \\
\hline & & Peso da dívida & 12 & \\
\hline & & Relação com a dívida estrutural & 6 & \\
\hline & \multirow[t]{4}{*}{ Independência } & Diversificação produtiva & 10 & \multirow[t]{4}{*}{25} \\
\hline & & Diversificação de relações contratuais & 10 & \\
\hline & & Conscientização de ajuda & 6 & \\
\hline & & Contribuição de renda externa para a independência & 4 & \\
\hline & \multirow[t]{2}{*}{ Transmissibilidade } & Transmissibilidade econômica & 15 & \multirow[t]{2}{*}{20} \\
\hline & & Sustentabilidade provável & 8 & \\
\hline & \multirow[t]{2}{*}{ Eficiência geral } & Eficiência bruta do processo de produção & 12 & \multirow[t]{2}{*}{20} \\
\hline & & Sobriedade no uso de insumos no processo produtivo & 8 & \\
\hline & \multicolumn{2}{|r|}{ Total } & 111 & 100 \\
\hline
\end{tabular}

Fonte: Adaptado de Zahm et al. (2015).

\section{RESULTADOS E DISCUSSÃO}

Ao avaliar a sustentabilidade de AG1, AG2, AG3, AG4 e AG5, nota-se resultados parecidos (Figura 2). Nestas, a dimensão Agroecológica obteve o melhor desempenho, evidenciando o bom uso das práticas agrícolas e os cuidados com o meio ambiente. Com exceção de AG2, a dimensão Econômica foi a que obteve o menor valor. De forma geral, o desenvolvimento sustentável dos agricultores familiares é comprometido muitas vezes por presença de dívidas e pela falta de capital financeiro, fator este que contribui para garantir a implementação das boas práticas, qualidade de vida e o desejo de continuar com o manejo agroecológico. Já o resultado da dimensão Sócio-territorial foi bastante equilibrado, com valores entre 70 e 83 . Esta dimensão não foi a que mais se destacou, porém apresentou um padrão consolidado, o que leva à compreensão da presença de um comportamento cultural estável nestas propriedades.

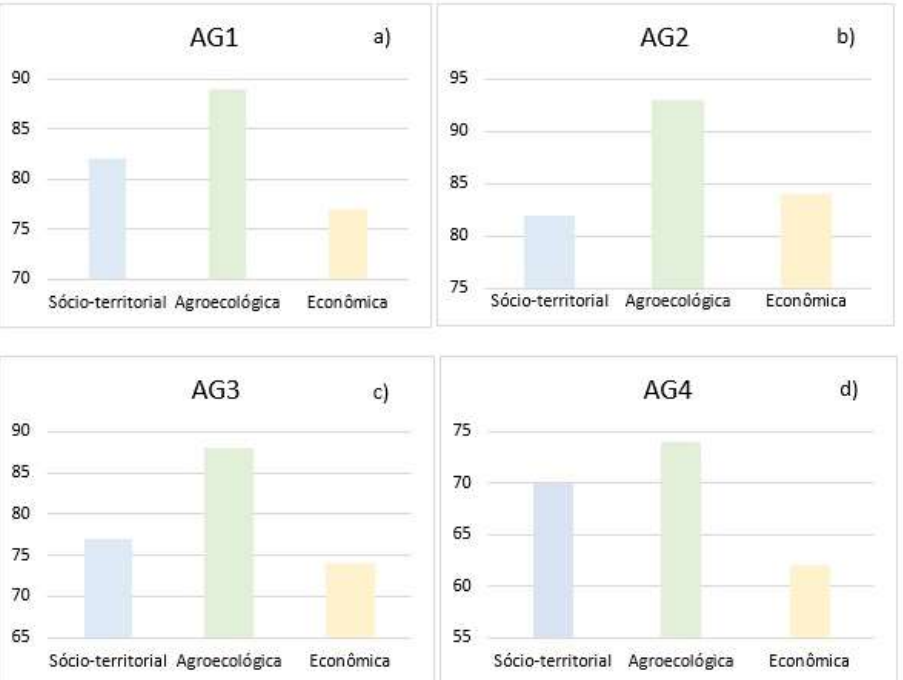

Figura 2: Pontuação de sustentabilidade referentes às dimensões Sócio-territorial, Agroecológica e Econômica das seguintes propriedades: a) AG1; b) AG2; c) AG3; d) AG4.

Os resultados das três dimensões (Sócio-territorial, Agroecológica e Econômica) para AG5, AG6, AG7, AG8, AG9 e AG10 descritos na figura 3 confirmam que, de modo geral, os cuidados ambientais estão ocorrendo nas propriedades. O desempenho dessa dimensão só não foi o melhor em AG7, onde 
sobressaíram as práticas culturais presentes na dimensão Sócio-territorial. Estes resultados também corroboram que a dimensão Econômica é o maior empecilho que os agricultores têm enfrentado. Assim, entende-se que, para alcançar maiores valores de sustentabilidade, se faz necessário o estímulo de práticas como a gestão dos dados financeiros, diversificação produtiva e auxílio de políticas públicas voltadas ao acesso e garantia de crédito rural.

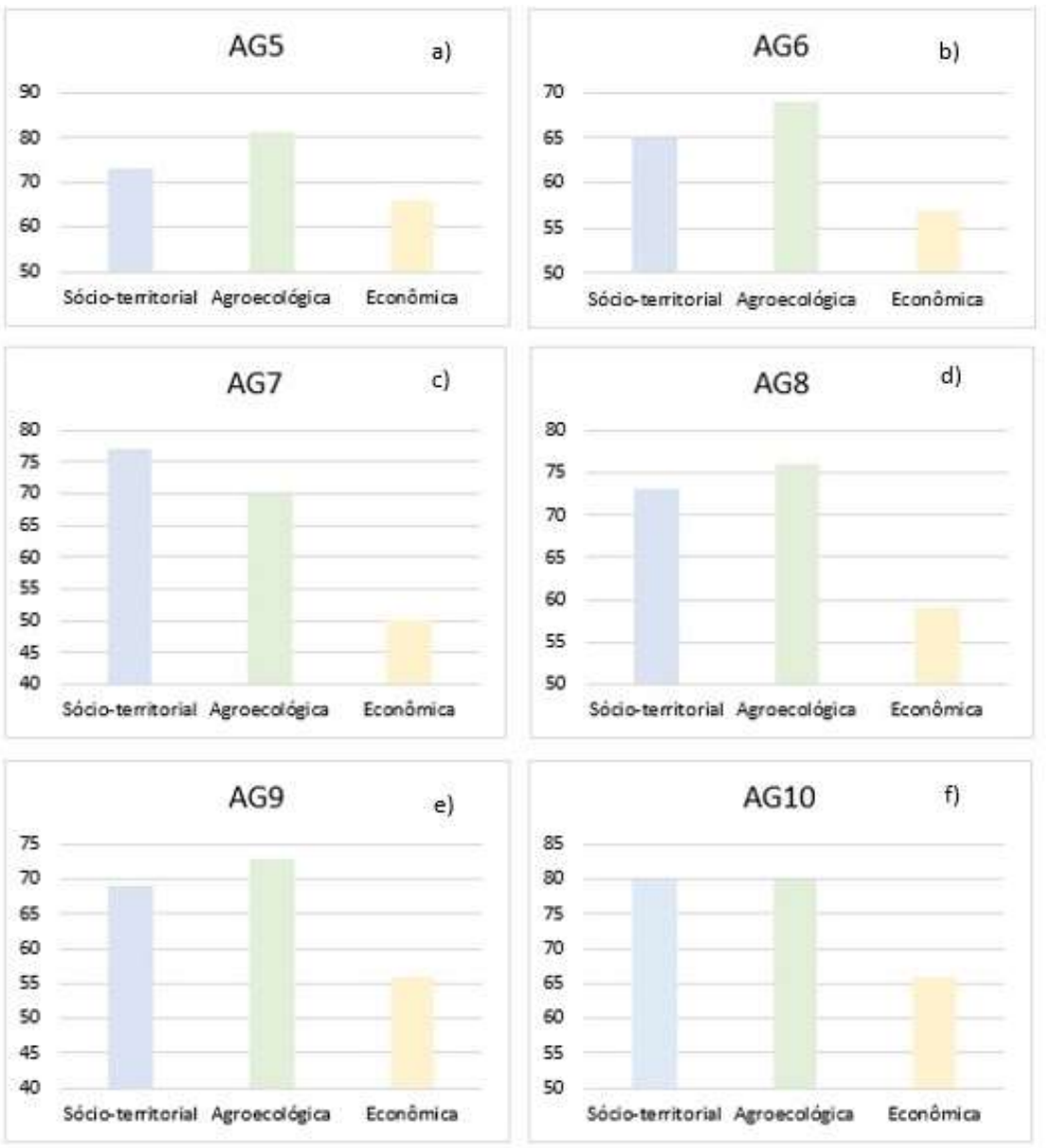

Figura 3: Pontuação de sustentabilidade referentes às Dimensões Sócio-territorial, Agroecológica e Econômica das seguintes propriedades: a) AG5; b) AG6; c) AG7; d) AG8; e) AG9; f) AG10.

De modo geral, os agroecossistemas avaliados apresentaram uma média de $74,8 \%$ para dimensão Sócio-territorial, 79,3\% para dimensão Agroecológica e 65,1\% para dimensão Econômica. Portanto, a dimensão Agroecológica foi a mais promissora e a dimensão Econômica a que mais prejudicou na sustentabilidade. As propriedades estudadas são pertencentes a agricultores que comercializam seus produtos em uma feira agroecológica, na qual dispõe de declaração de cadastro no Cadastro Nacional de Produtores Orgânicos, do Ministério da Agricultura, Pecuária e Abastecimento (MAPA). São agricultores acompanhados pelo Núcleo Agrofamiliar, que é vinculado à Universidade Federal do Agreste de Pernambuco (UFAPE), e por meio de professores e estudantes de graduação e pós graduação, desenvolve atividades de assessoria e acompanhamento, com realização de oficinas, cursos, intercâmbios e orientações técnico-produtivas. Portanto, esses fatores podem ter colaborado com o bom funcionamento das práticas voltadas ao eixo Agroecológico. A identificação do eixo de maior e menor pontuação é útil para apontar o fator que mais tem limitado e o que mais tem colaborado com a sustentabilidade e é sobre a 
dimensão com pior resultado que devem se voltar as principais práticas corretivas (VILAIN et al., 2008).

Ao comparar com outros estudos que utilizaram o mesmo método, verificou-se resultados diferentes. Melo et al. (2013) ao avaliarem a sustentabilidade de 10 propriedades agrofamiliares no Município de Ceará-Mirim (Rio Grande do Norte) observaram que a dimensão Econômica (68\%) foi a mais positiva e Sócio-territorial (48\%) a mais negativa. Já Silva (2019) avaliou a sustentabilidade de 39 propriedades familiares em Lagarto (Sergipe), e identificou que o fator limitante foi o eixo Agroambiental com $42 \%$ e o fator que mais favoreceu foi o eixo Econômico com $66,1 \%$. Embora exista divergências entre os resultados de trabalhos voltados a avaliação da sustentabilidade de propriedades familiares, os dados obtidos neste estudo mostram um nível de sustentabilidade acima da média, tendo em vista que as três dimensões estiveram acima de 50\%. A melhor performance relativa à sustentabilidade se deu especialmente aos aspectos agroambientais, e essa configuração já era de certo modo esperada, haja vista a proposta ecológica embutida nestes agroecossistemas.

Na pontuação geral para dimensão Sócio-territorial, onde a pontuação máxima é de 118, AG1, AG2 e AG10 atingiram os maiores valores com 82, 82 e 80 respectivamente. Já AG6 (65/118) e AG9 (69/118) apresentaram os valores mais baixos. Se tratando dos resultados das componentes que constituem esta dimensão, AG1 obteve maior valor em Desenvolvimento local e economia circular (21/25) e Ética e desenvolvimento humano (23/25). Em AG2 e AG10, os destaques foram as componentes Alimentação e Ética e desenvolvimento humano, com 22 cada, valores ótimos para dimensão em questão, tendo em vista que, o teto de todas suas componentes era de 25 (Figura 4). De modo geral a componente Alimentação foi a que obteve os melhores resultados. Assim, as atividades produtivas dos agroecossistemas têm colaborado positivamente com a sustentabilidade. Já a componente Emprego e qualidade no trabalho foi a que menos se destacou, o que a coloca como o principal gargalo a ser enfrentado pelos produtores avaliados.

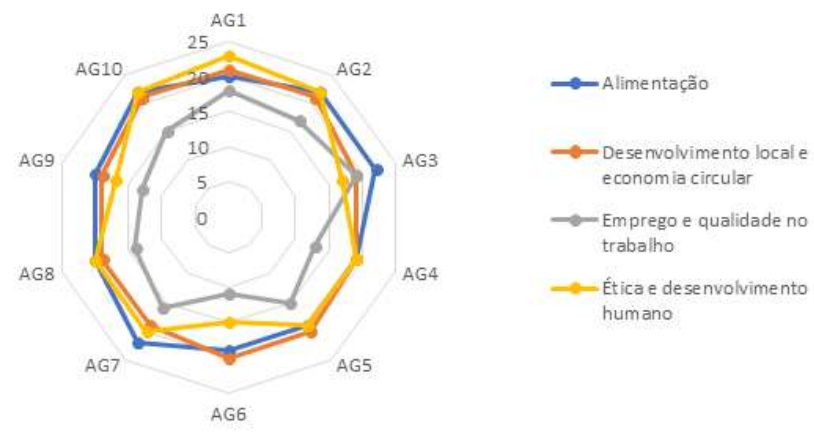

Figura 4: Resultados dos componentes da dimensão Sócio-territorial.

Em relação à dimensão Agroecológica, onde a pontuação máxima é de 121, as propriedades que apresentaram os melhores desempenhos foram: AG1 (89), AG2 (93) e AG3 (88). Já AG4, AG7, AG8 e AG9 apresentaram os menores valores, com 74, 70, 76 e 73, respectivamente. A componente Diversidade funcional foi a mais expressiva em AG1, no qual atingiu 20 pontos ( $100 \%$ do teto) e o menor desempenho foi em AG9, com 11 pontos. A componente Fluxo de materiais e energia através de uma busca por autonomia obteve destaque positivo em AG (19/20) e negativo em AG6 (11/20). Já a componente Garantia 
das condições favoráveis de produção a médio e longo prazo atingiu melhor resultado em AG2 (18/20), enquanto que, Sobriedade no uso dos recursos sobressaiu-se em AG1, AG2 e AG10, com 19 cada, valor próximo do teto (20) (Figura 5). De modo geral, a componente Sobriedade no uso dos recursos foi a que obteve o melhor desempenho. Este resultado deixa claro que os agricultores tem utilizado de forma racional os recursos naturais. Por outro lado, a componente Garantia das condições favoráveis de produção a médio e longo prazo foi a menos expressiva, o que demonstra a necessidade de melhoria no manejo agrícola.
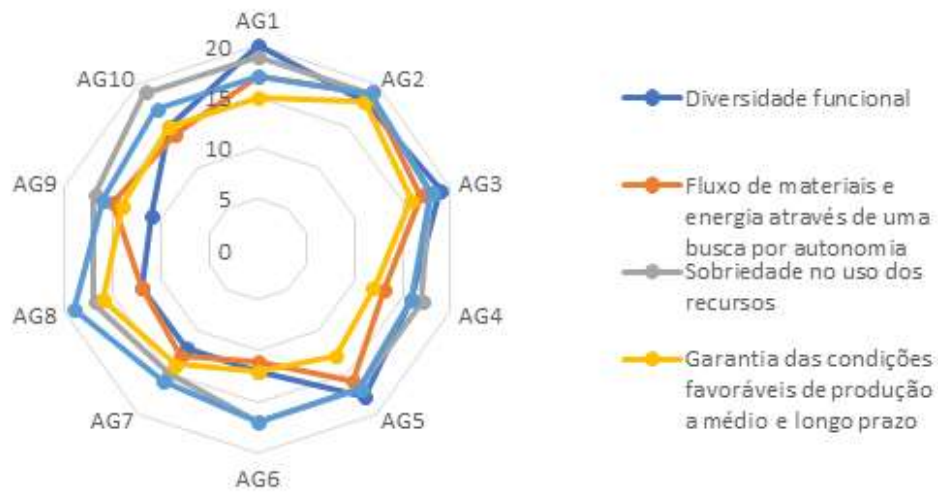

Figura 5: Resultados dos componentes da Dimensão Agroecológica.

Durante a avaliação da dimensão Econômica notou-se que AG2 atingiu a melhor pontuação, chegando a 84, enquanto que, AG7 pontuou apenas 50 dos 111. A componente Viabilidade econômica e financeira destacou-se positivamente em AG1 (26/35) e negativamente em AG7 (15/35). Independência atingiu seu teto em AG2 (25/25) e obteve os menores valores em AG4 e AG7, com 14 cada. Transmissibilidade que tinha o valor máximo de 20, pontuou positivamente em AG1 e AG2, ambas com 19, e negativamente em A7 e AG8, com 11 cada. Já a componente Eficiência geral obteve melhor nota em AG1 (17/20) e a menor em AG7 (10/20) (Figura 6). De modo geral, a componente Independência $(70,8 \%)$ foi a que mais contribui para o seu bom resultado. Assim, compreende-se que a diversificação da produção e a presença de renda extra têm colaborado positivamente. Já a componente Viabilidade econômica e financeira $(58,8 \%)$ foi a que mais prejudicou. A falta de capital financeiro para expansão das atividades e a presença de dívidas em alguns casos dificultam um melhor resultado desta dimensão.

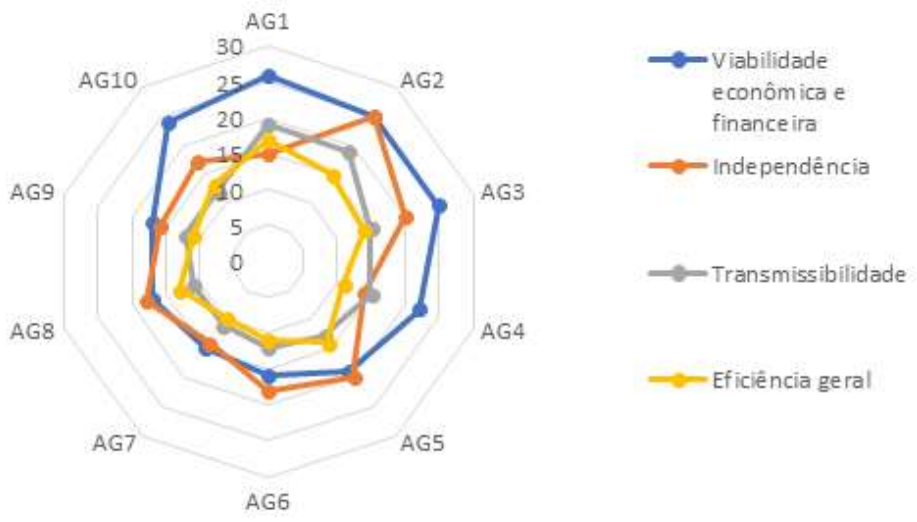

Figura 6: Resultados dos componentes da Dimensão Econômica. 
Este trabalho levou em consideração os novos desafios sociais (alimentos, mudanças climáticas, qualidade do ar, sobriedade no uso de recursos) e enriquecimento do ambiente teórico para analisar a sustentabilidade (princípios da agroecologia, economia circular, local de ação coletiva, sobriedade no processo produtivo). O método IDEA tem passado por várias adaptações e utilizações para o contexto da agricultura familiar em diversos estados do Brasil. Porém, para as avaliações de propriedades agrícolas brasileiras ainda carece de informações acerca dos graus de sustentabilidade agrícola e caraterísticas da agricultura familiar, portanto, este trabalho contribui com a dinâmica da operacionalização do método neste segmento (MELO, 2013). Ressalta-se ainda, a importância do uso do método IDEA v4 para avaliar a sustentabilidade dos agroecossistemas em questão, tendo em vista que, este pode levar a uma renovação de práticas, em especial no apoio à transição agroecológica (MANNEVILLE et al., 2018), bem como subsidiar planejamentos de gestão pública e de políticas alimentares.

\section{CONCLUSÕES}

Ao avaliar a sustentabilidade destas propriedades familiares esperava-se intuitivamente que as mesmas apresentem níveis médios ou altos de sustentabilidade, tendo em vista o envolvimento com as práticas agroecológicas. Entende-se que, quanto mais avançado o nível de transição agroecológica, também maior será a expectativa de sustentabilidade. Os resultados dos agroecossistemas mostrou que ambos obtiveram bons resultados na avaliação de sustentabilidade quando observados os eixos Agroambiental, Sócio-territorial e Econômico, sendo as melhores pontuações relativas aos aspectos ambientais e as piores ao fator econômico. Assim, chama-se atenção para a dimensão Econômica, tendo em vista a necessidade de melhorar algumas potencialidades e buscar reduzir as limitações ao longo do espaço e do tempo.

A aplicação do método IDEA v4 centrado nas três dimensões se mostrou eficiente para a captação da sustentabilidade de forma holística. A coleta de informações se deu de forma flexível e a apresentação de seus resultados é simples, onde é possível identificar os principais gargalos desses sistemas e suas potencialidades, o que auxilia na tomada de decisão tanto para as famílias dos agricultores quanto para assistência técnica e extensão Rural, considerando o diagnóstico das propriedades. No entanto, nota-se a falta de indicadores voltados a questão da equidade de gênero, o que enriqueceria ainda mais a ferramenta. As questões abordadas neste estudo almejam levantar um debate sobre o uso de indicadores de sustentabilidade, colaborando dessa forma, com futuros trabalhos. E contribuir com a operacionalização de ferramentas que colaborem com a autogestão de agroecossistemas, a sustentabilidade e tomada de decisão de gestores com subsídios para elaboração de políticas públicas.

\section{REFERÊNCIAS}

BRASIL. Plano Territorial do Desenvolvimento Rural Sustentável do Agreste Meridional de Pernambuco, 2011.

DE OLDE, E. M.; OUDSHOORN, F. W.; S $\varnothing$ RENSEN, C. A.; BOKKERS, E. A.; DE BOER, I. J.. Assessing sustainability at farm-level: lessons learned from a comparison of tools in practice. Ecological Indicators, v.66, p.391-404, 2016. DOI: https://doi.org/10.1016/j.ecolind.2016.01.047

HANSEN, J. W.. Is agricultural sustainability a useful concept? Agricultural systems, v.50, n.2, p.117-143, 1996. DOI: https://doi.org/10.1016/0308-521X(95)00011-s

KATES, R. W.; PARRIS, T. M.; LEISEROWITZ, A. A.. What is 
sustainable development. Environment: Science and Policy for Sustainable Development, v.47, n.3, p 8-21, 2005. DOI: https://doi.org/10.1080/00139157.2005.10524444

LAIREZ, J.; FESCHET, P.; AUBIN, J.; BOCKSTALLER, C.; BOUVAREL, I.. Évaluer la durabilité en agriculture. Guide pour l'analyse multicritère des productions animales et végétales. Dijon: éditions Éducagri, 2015.

LÓPEZ-RIDAURA, S.; MASERA, O.; ASTIER, M.. Evaluating the sustainability of complex socio-environmental systems. The MESMIS framework. Ecological indicators, v.2, n.1-2, p.135148, 2002. DOI: https://doi.org/10.1016/S1470$160 \times(02) 00043-2$

LÓPEZ-RIDAURA, S.; VAN KEULEN, H; VAN ITTERSUM, M. K.; LEFFELAAR, P. A.. Multiscale methodological framework to derive criteria and indicators for sustainability evaluation of peasant natural resource management systems. Environment, development and sustainability, v. 7, n.1, p.51-69, 2005. DOI: https://doi.org/10.1007/s10668003-6976-x

MANNEVILLE, V.; GIRARD, S.; ZAHM, F.. Les propriétés de la durabilité en agriculture pour préparer la transition agroécologique face à l'enjeu du changement climatique. 2018.

MELO, L. E. L.; CÂNDIDO, G. A.. O uso do método IDEA na avaliação de sustentabilidade da agricultura familiar no município de Ceará-Mirim - RN. Revista de Administração, Contabilidade e Sustentabilidade, v.3, n.2, p.1-19, 2013.

POPE, J.; ANNANDALE, D.; MORRISON-SAUNDERS, A. Conceptualising sustainability assessment. Environmental impact assessment review, v.24, n.6, p.595-616, 2004. DOI: https://doi.org/10.1016/j.eiar.2004.03.001

PINSONNEAULT, A.; KRAEMER, K. L.. Survey research in management information systems: an assessement. Journal of Management Information System, v.10, 1993.

PUJOL, J. L.; DRON, D.. Agriculture, monde rural et environnement: qualité oblige. Le Courrier de l'environnement de l'INRA, v.37, n.37, p.52-56, 1999.

SCHADER, C.; GRENZ, J.; MEIER, M. S.; STOLZE, M.. Scope and precision of sustainability assessment approaches to food systems. Ecology and society, v.19, n.3, 2014. DOI: http://dx.doi.org/10.5751/ES-06866-190342

SILVA, D. S. C.. Aspectos da sustentabilidade de explorações fumageiras no Centro - Sul de Sergipe. Dissertação (Mestrado em Desenvolvimento e Meio Ambiente) Universidade Federal de Sergipe, São Cristóvão, 2019.

TAUBER, M.. Pressions et services environnementaux. L'agriculture, nouveaux défis. p.178190, 2007.

VILAIN, L.; BOISSET, K.; GIRARDIN, P.; GUILLAUMIN, A. MOUCHET, C.; VIAUX, P.; ZAHM, F.. La méthode IDEA Indicateurs de durabilité des exploitations agricoles. Dijon: Educagri, 2008

ZAHM, F.; UGAGLIA, A. A.; BOUREAU, H.; DEL'HOMME, B.; BARBIER, J. M.; GASSELIN, P.; GAFSI, M.; GUICHARD, L.; LOYCE, C.; MANNEVILLE, V.; MENET, A.; REDLINGSHOFER.. Agriculture et exploitation agricole durables: état de l'art et proposition de définitions revisitées à l'aune des valeurs, des propriétés et des frontières de la durabilité en agriculture. Innovations Agronomiques, n.46, p.105-125, 2015. DOI:

https://dx.doi.org/10.15454/1.462267509242779E12

ZAHM, F.; UGAGLIA, A. A.; BARBIER, J. M.; BOUREAU, H.; DEL'HOMME, B.; GAFSI, M.; GASSELIN, P.; GIRARD, S.; GUICHARD, L.; LOYCE, C.; MANNEVILLE, V.; MENET, A.; REDLINGSHÖFER, B.. Evaluating sustainability of farms: introducing a new conceptual framework based on three dimensions and five key properties relating to the sustainability of agriculture. In: EUROPEAN IFSA SYMPOSIUM, 13. Procedures. Chania, 2018.

ZAHM, F.; UGAGLIA, A. A.; BARBIER, J. M.; BOUREAU, H.; DEL'HOMME, D.; GAFSI, M.; GASSELIN, P.; GIRARD, S.; GUICHARD, L.; LOYCE, C.; MANNEVILLE, V.; MENET, A.; REDLINGSHÖFER, B.. Évaluer la durabilité des exploitations agricoles: la méthode IDEA v4, un cadre conceptuel combinant dimensions et propriétés de la durabilité. Cahiers Agricultures, v.28, n.5, p.10, 2019. DOI: https://doi.org/10.1051/cagri/2019004

A CBPC - Companhia Brasileira de Produção Científica (CNPJ: 11.221.422/0001-03) detém os direitos materiais desta publicação. Os direitos referem-se à publicação do trabalho em qualquer parte do mundo, incluindo os direitos às renovações, expansões e disseminações da contribuição, bem como outros direitos subsidiários. Todos os trabalhos publicados eletronicamente poderão posteriormente ser publicados em coletâneas impressas sob coordenação da Sustenere Publishing, da Companhia Brasileira de Produção Científica e seus parceiros autorizados. Os (as) autores (as) preservam os direitos autorais, mas não têm permissão para a publicação da contribuição em outro meio, impresso ou digital, em português ou em tradução. 